\title{
Swept Confocally-Aligned Planar Excitation (SCAPE) Microscopy for High Speed Volumetric Imaging in Behaving Animals
}

Elizabeth M. C. Hillman ${ }^{1,2}$, Matthew B. Bouchard ${ }^{1}$, Venkatakaushik Voleti ${ }^{1}$, Wenze Li ${ }^{1}$, César S. $^{4}$ Mendes $^{3}$, Clay Lacefield ${ }^{4}$, Vanessa George ${ }^{5}$, Wesley B. Grueber ${ }^{6}$, Richard S. Mann ${ }^{3}$, Randy M. Bruno $^{2,4}$, Kimara Targoff ${ }^{5}$.

${ }^{1}$ Laboratory for Functional Optical Imaging, Departments of Biomedical Engineering and Radiology*

${ }^{2}$ Kavli Institute for Brain Science*

${ }^{3}$ Mann Lab, Department of Biochemistry and Molecular Biophysics*

${ }^{4}$ Bruno Lab, Department of Neuroscience*

${ }^{5}$ ZFIN Lab, Department of Pediatrics**

${ }^{5}$ Department of Physiology and Cellular Biophysics, Department of Neuroscience**

*Columbia University, **Columbia University Medical Center, New York, New York 10027/32, USA,

New contrast agents and genetic techniques have delivered complex organisms and in-vivo tissues painted with a palette of dynamically changing fluorescence [1]. These tissues are inherently threedimensional, yet methods to image volumes of tissue at the microscopic scale face many problems that make capturing high-speed dynamics a major challenge. Laser scanning confocal and two-photon imaging are generally limited by their need to serially visit single points within the volume. As a result, these modalities are almost always used to image a single plane rather than a volume of tissue if high acquisition speeds are required, causing them to miss information beyond the plane, and to be highly sensitive to sample movement [2]. Moreover, when 3D scanning is used, the standard method is to physically translate the objective lens up and down to sample different planes, a process that slows acquisition, increases the risk of mechanical failure and can disturb the living sample being imaged.

Light-sheet microscopy benefits from parallel imaging of an entire plane of the sample in a single camera frame, achieving optical sectioning through the use of a thin sheet of light for illumination [3]. However, typical implementations of this approach use two orthogonally-aligned objective lenses, one to generate the light sheet and one to image it. This restricts the shape and size of the sample that can be imaged, while also imposing the need to translate the sample or the objective assembly relative to one another in order to generate a 3D image. In practice, this need for relative translation limits volumetric imaging speed and imposes the need to physically mount, hold and sometimes translate the sample [3].

Swept Confocally-Aligned Planar Excitation (SCAPE) microscopy is a new approach to fast 3D microscopy that overcomes many of the limitations of the techniques described above [4]. Firstly, SCAPE uses light-sheet illumination, but delivers it to the sample at an oblique angle through the same objective lens as is used to detect the resultant fluorescence, overcoming the need for two objective lenses (see Figure 1). Secondly, SCAPE sweeps this light sheet laterally (along x') using a scan mirror, as in confocal line-scanning microscopy, in order to sample a full volume of the tissue as a series of y'z' planes. Finally, SCAPE reflects the emitted fluorescence light off the same scan mirror (in our first prototype, an adjacent facet of a polygonal mirror mounted on a galvanometer motor), causing the light to be de-scanned as in confocal microscopy. The result is an oblique image plane that is stationary, and corresponds to an image of the tissue that is being optically sectioned by the sweeping oblique light sheet - the detection plane stays aligned with light sheet as the sheet moves. Image rotation optics map this stationary image plane onto a free-running high-speed camera (Andor Zyla-5.5-CL10) which can grab 2,560 x 80 (y'-z') planes at 2,400 fps. Since one sweep of the mirror corresponds to acquisition of an entire volume, there are no physical limits to acquisition speed except camera frame rate and signal to 
noise: so a 200 or 100 (x') voxel wide, 80 layer deep volume can be acquired at 12 or 24 volumes per second respectively. Importantly, this volumetric imaging is achieved with no physical translation of the objective lens or sample, making SCAPE capable of imaging almost any un-mounted sample in an enface imaging geometry, with no concern for physical motion of components interfering with the sample or limiting imaging speed. SCAPE is currently implemented with a $488 \mathrm{~nm}$ DPSS CW laser source.

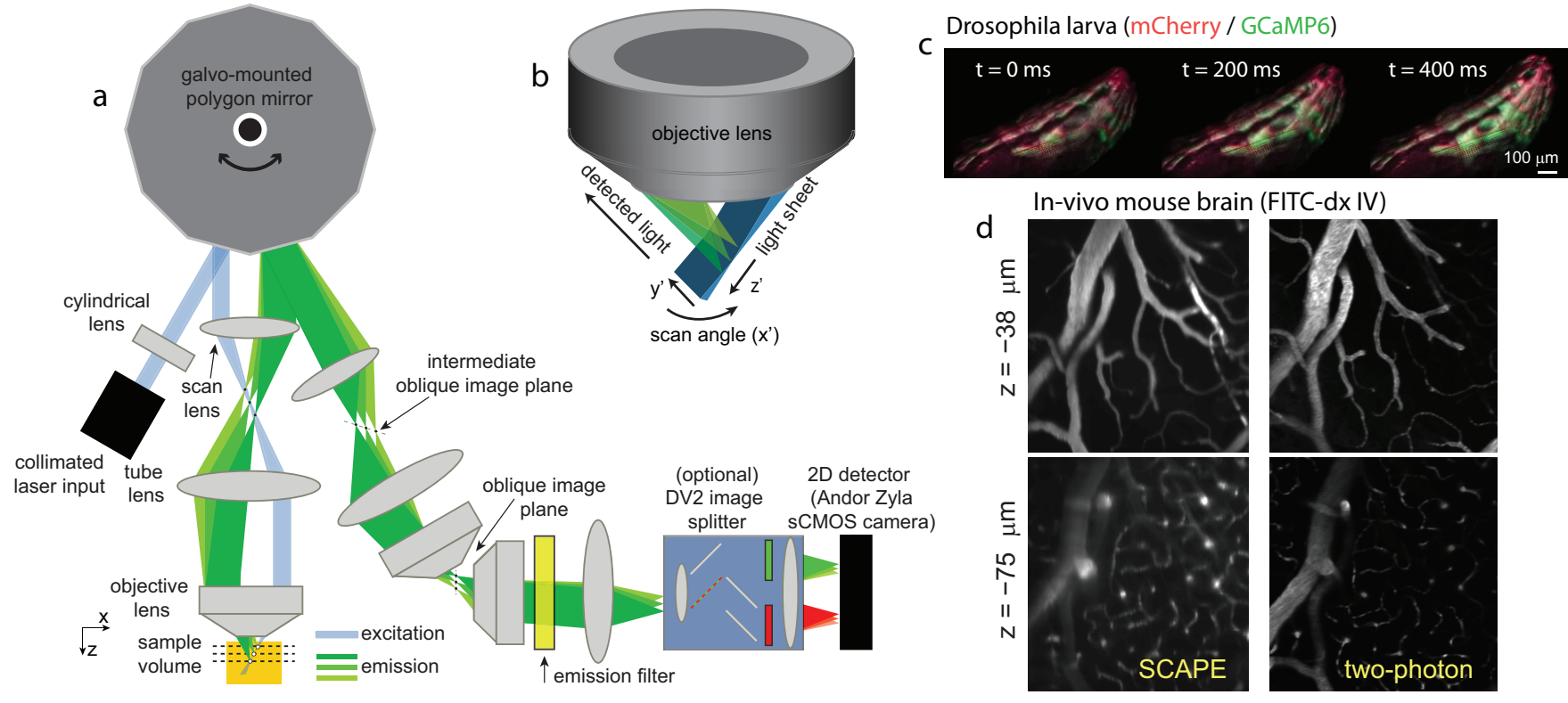

Figure 1. SCAPE prototype and results. A polygon mirror is swept back and forth at the volume rate. This scanning causes the oblique sheet of light (blue) formed in the y'-z' plane of sample to sweep back and forth along $\mathrm{x}$ '. The light emerging from the sample (green) is descanned from the same moving mirror, causing the oblique image produced to remain stationary, but to correspond to the moving plane illuminated in the sample. Image rotation optics and a (removable) dual-color image splitter project this image onto the face of a fast camera. (c-d) Examples of SCAPE microscopy in living organisms. c) shows a time-sequence of rendered SCAPE data acquired on a freely moving first instar Drosophila melanogaster (fruit fly) larva expressing mCherry and GCaMP6 in its muscles (lexO-GcaMP6f;mhclexA,lexO-mCherry::CAAX) at 10 volumes per second (VPS) during a 'head-lift'. d) Shows a comparison between SCAPE and two-photon microscopy of vasculature in living mouse brain. SCAPE images of neural activity in superficial dendrites have been recorded at 10 VPS in awake, behaving mice. See [4] for more details and results.

Acknowledgments / conflict of interest: Kavli Foundation, NIH (NINDS) R21NS053684, R01 NS076628 and R01NS063226, NSF CAREER 0954796, DoD MURI W911NF-12-1-0594, HFSP, Wallace H. Coulter Foundation, NSF BioIGERT (OTASI), NSF GRFP and NDSEG. Conflict of interest: A patent on this technology (US20120140240) is held by the Trustees of Columbia.

1. Akerboom, J., et al., Genetically encoded calcium indicators for multi-color neural activity imaging and combination with optogenetics. Frontiers in Molecular Neuroscience, 2013. 6.

2. Cotton, R.J., et al., Three-dimensional mapping of microcircuit correlation structure. Front Neural Circuits, 2013. 7: p. 151.

3. Ahrens, M.B., et al., Whole-brain functional imaging at cellular resolution using light-sheet microscopy. Nature Methods, 2013. 10(5): p. 413-20.

4. Bouchard, M.B., et al., Swept confocally-aligned planar excitation (SCAPE) microscopy for highspeed volumetric imaging of behaving organisms. Nature Photonics, 2015. 9(2): p. 113-119. 